Research Article

\title{
Effect of Micro- and Nanoparticles of Ampicillin Trihydrate on Blood Medium: A Voltammetric Study
}

\author{
Muhammed Mizher Radhi ${ }^{1}$, Zuhair Numan Hamed ${ }^{2}$, Selda Sabah Ezzaldeen², \\ Emad Abbas Jaffar Al-Mulla \\ ${ }^{1}$ Radiological Techniques Department, Health and Medical Technology College-Baghdad, Middle Technical University (MTU), Iraq. \\ ${ }^{2}$ Lab. Techniques Department, Health and Medical Technology College-Baghdad, Middle Technical University (MTU), Iraq. \\ ${ }^{3}$ College of Health and Medical Techniques, Al-Furat Al-Awsat Technical University, 54003 Al-Kufa, Iraq. \\ Corresponding authors. E-mail: mmrradhi@yahoo.com; emadalmulla@atu.edu.iq
}

Received: Jun. 27, 2017; Accepted: Aug. 9, 2017; Published: Aug. 11, 2017.

Citation: Muhammed Mizher Radhi, Zuhair Numan Hamed, Selda Sabah Ezzaldeen, and Emad Abbas Jaffar Al-Mulla, Effect of Micro- and Nanoparticles of Ampicillin Trihydrate on Blood Medium: A Voltammetric Study. Nano Biomed. Eng., 2017, 9(3): I85-190. DOI: 10.5101/nbe.v9i3.p185-190.

\begin{abstract}
For the first time, one of the antibiotic nanoparticles such as a classical form of ampicillin trihydrate compound was studied. The electrochemical behavior of ampicillin nanoparticles was investigated in blood medium using cyclic voltammetric technique by glassy carbon electrode. The results showed that the oxidation-reduction current peaks of ampicillin nanoparticles in blood medium were different from that of microparticles. The nanoparticles acted as anti-oxidative antibiotic by making the oxidation current peak at $1 \mathrm{~V}$ disappear, while the oxidation peak of microparticles still appeared in blood medium; hence, ampicillin at microform acted as oxidative reagent in blood medium. A good reliability and stability of glassy carbon electrode in blood medium was found with low values of RSD for oxidation-reduction current peaks at $\pm 0.52 \%$ and $\pm 0.038 \%$ respectively. Scanning electron microscopy for the characterization of ampicillin trihydrate nanoparticles was studied.
\end{abstract}

Keywords: Ampicillin trihydrate; Cyclic voltammetry; Nanoparticles; Blood medium; Redox process

\section{Introduction}

Cyclic voltammetric technique was used by scientists to identify the impact of chemical compounds on the blood components during the exposure of the body to pollutants or drugs [1-6]. Recent studies have confirmed that antibiotic compounds after being converted to nanoparticles showed great effect on the elimination of different types of bacteria which cause diseases [7].

Studies have shown that silver nanoparticles (AgNPs) were intrinsically antibacterial, but gold nanoparticles (AuNPs) were antimicrobial only when ampicillin was bound to their surfaces. Both AuNPs and AgNPs functionalized with ampicillin were effective broad-spectrum bactericides against Gramnegative and Gram-positive bacteria. Most importantly, when AuNPs and AgNPs were functionalized with ampicillin, they became potent bactericidal agents with unique properties that subverted antibiotic resistance mechanisms of multiple-drug-resistant bacteria [8].

The physical chemistry properties of negatively charged liposomes and of poly-iso-hexyl-cyanoacrylate nanoparticles were loaded with ampicillin. Although 
the carriers were of the same size $(200 \mathrm{~nm})$, drugloading capacity was 20 times higher for nanoparticles than for liposomes. Drug release in fetal calf serum was gradual (of zero order) with nanoparticles, whereas it was rapid with liposomes, the antimicrobial activity of ampicillin-entrapped liposomes or nanoparticles was studied in vitro [9].

Poly-iso-hexyl-cyanoacrylate nanoparticles were studied in vitro in mouse peritoneal macrophages infected with Listeria monocytogenes. Nanoparticles containing ampicillin $1 \mathrm{mg} / \mathrm{L}$ were more effective after $30 \mathrm{~h}$ than free ampicillin at the same concentration. The empty nanoparticles had neither an anti-listeria nor a cytotoxic effect [10].

A new method of supercritical gas anti-solvent (GAS) reaction was carried out for ampicillin nanoparticles synthesis. The particle size distribution of ampicillin limits bioavailability. The effect of antisolvent addition rate on nucleation indicated that nucleation was enhanced via higher anti-solvent addition rate and consequently smaller particle size was obtained. The mean particle sizes of ampicillin were obtained to be $357.09,337.04$ and $356.68 \mathrm{~nm}$ at anti-solvent flow rates of $1.6,2$ and $2.4 \mathrm{~mL} / \mathrm{min}$, respectively [10].

Ampicillin trihydrate-loaded chitosan nanoparticles were prepared by ionic gelation method with the aid of sonication. The antibacterial properties of the nanoparticle formulation were evaluated and compared with that of a commercial formulation. The modification of ionic gelation method can be utilized for the development of chitosan nanoparticles of ampicillin trihydrate [12].

Four formulations of ampicillin were formulated using different drug polymer ratios. The formulation is subjected to evaluation parameters like particle size, zeta potential, drug content uniformity, percentage yield, entrapment efficiency, drug loading efficiency in-vitro drug released study [13].

In this study, ampicillin nanoparticles were prepared by different two methods from ampicillin microparticles and study the electrochemical behavior in blood medium using cyclic voltammetric technique by glassy carbon electrode (GCE).

\section{Experimental}

\section{Preparation of ampicillin trihydrate nano-particles}

Two methods were used for the preparation of ampicillin trihydrate nanoparticles from the microparticles in the laboratory:

\section{Lyophylization (freeze-drying method)}

Ampicillin nanoparticles as prepared by dissolving $0.75 \mathrm{~g}$ of ampicillin microparticles in $150 \mathrm{~mL}$ of distilled water. The suspension of ampicillin trihydrate was cooled, and the ice crystals of pure water which formed at $-18{ }^{\circ} \mathrm{C}$. The second step involves the sublimation of ice from the frozen product by passing heat air from shelf of lyophylization instrument to the frozen solution in the vial, the ice sublimes and the water vapor formed passes through the dried portion of the product to the surface of the sample, the water vapor is transferred from the surface of the product through the chamber to the condenser, and the water vapor condenses on the condenser. At the end of sublimation step a porous plug is formed. Its pores correspond to the spaces that were occupied by ice crystals. The third step is drying involve the removal of absorbed water from the product. All steps must be continuous about $48-72 \mathrm{~h}$ [14].

\section{Reduction method by silver nitrate}

The another method of preparation ampicillin nanoparticles was prepared $200 \mathrm{~mL}$ of $1 \mathrm{mM}$ of silver nitrate solution $\left(\mathrm{AgNO}_{3}\right)$ by dissolve 0.169

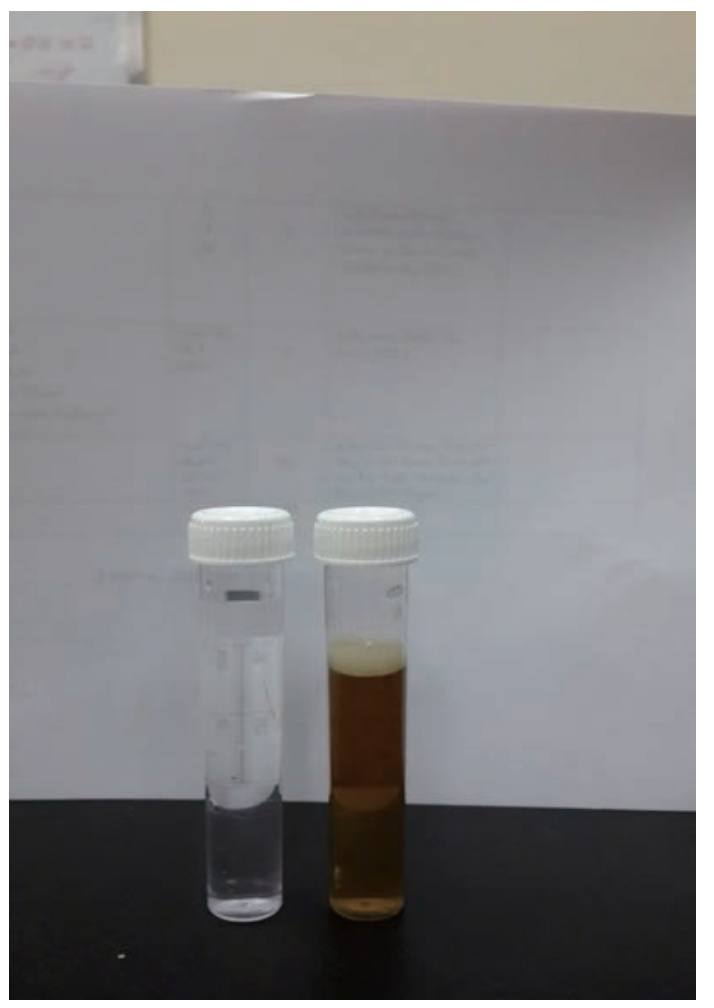

Fig. 1 Ampicillin trihydrate micro- and nanoparticles solution. 
gm of $\mathrm{AgNO}_{3}$ crystal in $200 \mathrm{~mL}$ of distilled water, the solution was kept in a $250 \mathrm{~mL}$ of reagent bottle. $0.025 \mathrm{gm}$ of ampicillin powder was dissolved in 1 $\mathrm{mL}$ of phosphate buffer and then added to $20 \mathrm{~mL}$ of the prepared silver nitrate solution. The $95 \%$ of bioreduction of $\mathrm{AgNO}_{3}$ ions occurred within $24 \mathrm{~h}$ to reducing ampicillin particles to nanoparticles as shown in Fig. 1(b). The deep yellowish solution in Fig. 1 for the ampicillin particles in phosphate buffer which turned into colorless slowly indicated the formation of ampicillin nanoparticles [15].

\section{Materials}

Ampicillin trihydrate compound form as white powder was bought from Bioanalyse Company (Turkey) and blood samples were extracted from healthy humans which received from the center medicine of Baghdad City was used in the analysis. Deionize water was used for the preparation of aqueous solutions. All blood samples were diluted with distilled water by ratio $1: 9 \mathrm{~mL}$ (blood: water), $10 \mathrm{~mL}$ of diluted blood was used in the cyclic voltammetric cell.

\section{Apparatus}

\section{Cyclic voltammetric technique}

The instrument EZstat series (Potentiostat/ Glvanostat) of NuVant Systems Inc. (made in USA). The Electrochemical bio-analytical cell was connected with potentio-state device and monitoring through the special program that has been installed on the personal computer to perform cyclic voltammetry (CV). The silver-silver chloride reference electrode $(\mathrm{Ag} / \mathrm{AgCl}$ in $3 \mathrm{M} \mathrm{NaCl})$ and platinum wire (1 $\mathrm{mm}$ diameter) was used as a reference and counter electrodes respectively. The glassy carbon working electrode (GCE) was used in this study after cleaning by polishing with alumina solution and treated with ultrasonic water path for ten minutes to performing the measurment.

\section{Lyophylization instrument}

Lyophylization instrument from LABCONCO Company (U.S.A.) was used to preparation of ampicillin trihydrate nanoparticles from microparticles by deep freezing technique as shown in Fig. 2 .

\section{Results and Discussion}

Ampicillin trihydrate compound in microstructure illustrated in scheme 1 was used at both microand nanoparticles in blood medium to study the electrochemical behavior at different concentrations

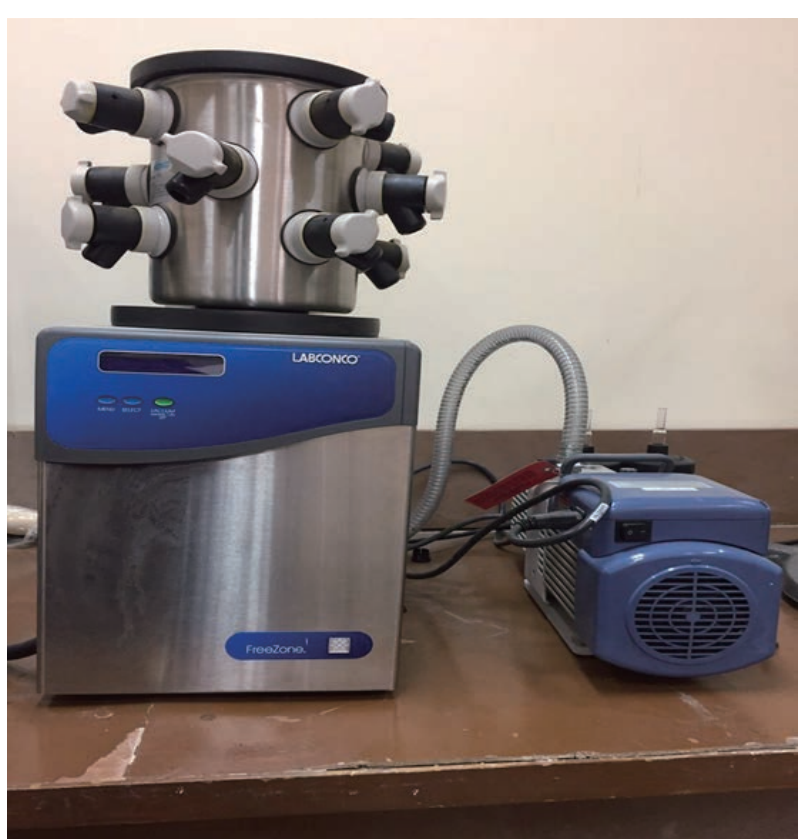

Fig. 2 Lyophylization instrument from LABCONCO Company (U.S.A.).

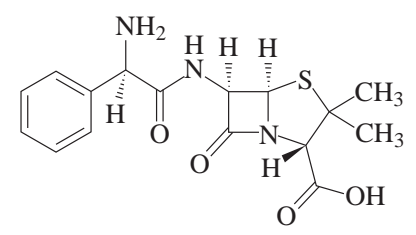

Scheme 1 Chemical structure of ampicillin trihydrate microparticles [16].

and scan rate, also the influence of different concentrations of ascorbic acid (AA) on redox current peaks of ampicillin in blood medium was studied.

\section{Redox effect of ampicillin trihydrate at micro- and nanoparticles in blood medium}

The electrochemical behavior of the ampicillin nanoparticles was found from the cyclic voltammetric analysis as antioxidative reagent in blood medium with the reduction current peak appearing at $-750 \mathrm{mV}$ as shown in Fig. 3, while the cyclic voltammogram of ampicillin at microparticles in blood medium showed the oxidation and reduction current peaks at +1 and $+0.75 \mathrm{~V}$ respectively; hence, the ampicillin at microparticles acted as an oxidative reagent in blood medium; it can be used the ampicillin nanoparticles as a safety and active antibiotic drug for different bacterial disease as anti-oxidative compound to the blood components [17].

\section{Effects of different concentrations of ampicillin trihydrate nanoparticles in blood medium}

The results of this study revealed that with 


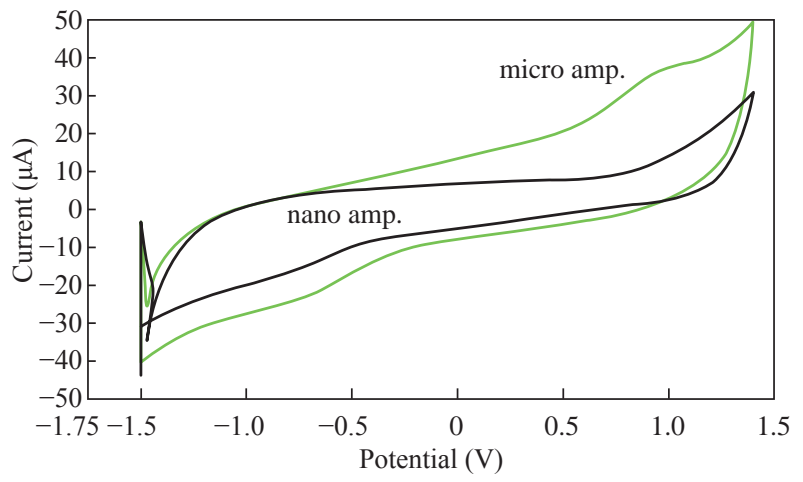

Fig. 3 Cyclic voltammogram of ampicillin trihydrate microand nanoparticles in blood medium at GCE as working electrode and $\mathrm{Ag} / \mathrm{AgCl}$ as reference electrode.

the increasing of the concentration of ampicillin nanoparticles in blood medium which acted as electrocatalyst of cathodic current peak as shown in Fig. 4 , there existed a linear relationship between the reduction current against different concentrations of nano-ampicillin as nano-antibiotic. It was found from the results that nanoparticles of the antibiotic drug such as ampicillin trihydrate acted as a good anti-oxidative in blood medium, while ampicillin in microform was found in electro-analysis as an oxidative anti-biotic because of its anodic current peak at $+1 \mathrm{~V}$ in blood medium which enhanced against the increasing of the concentration [18]. Fig. 4 showed a good linear relationship between the reduction current peak of ampicillin nanoparticles in different concentrations with high value of sensitivity $\left(R^{2}=0.912\right)$.

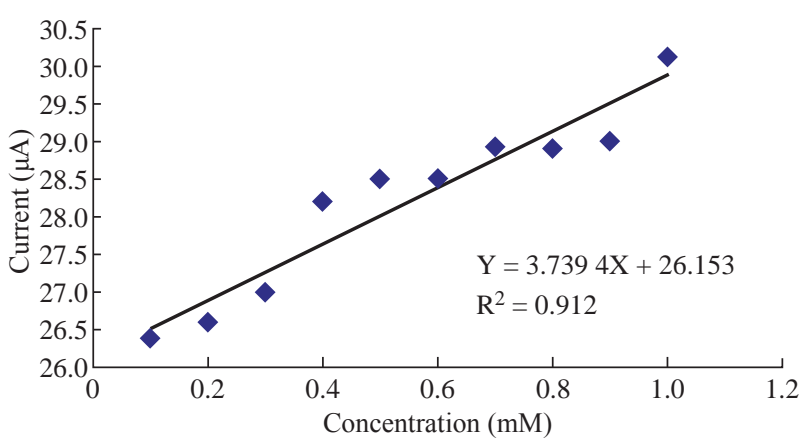

Fig. 4 Effects of different concentrations of ampicillin trihydrate nanoparticles in blood medium on cathodic current peak at GCE.

\section{Effects of different scan rates on the reduction peak of ampicillin nanoparticles in blood medium}

The effects of different scan rates were studied using GCE in blood medium to find the cathodic current peak of ampicillin nanoparticles at potential $-0.75 \mathrm{~V}$. A linear line of the relationship between cathodic current peak and different scan rates was plotted in Fig. 5 by the equation: $\mathrm{Y}=186.43 \mathrm{X}+16.607$ with good a sensitivity of $R^{2}=0.937$ [19].

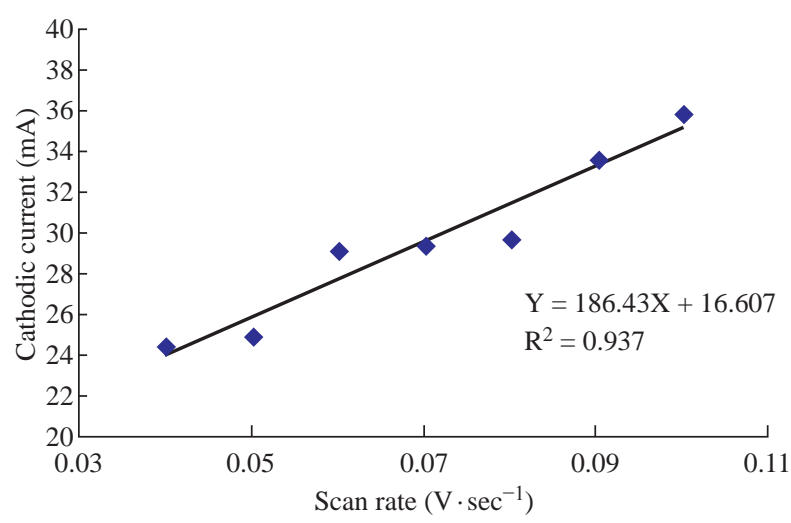

Fig. 5 Plot cathodic current peak of ampicillin nanoparticles in blood medium against to different scan rate using GCE versus $\mathrm{Ag} / \mathrm{AgCl}$.

\section{Reliability and stability of GCE in blood medium}

The potential cycling of the redox current peaks of GCE in blood medium was carried out during cyclic voltammetry. Continuous potential cycling did not seem to affect the redox current peak of the GCE in blood medium, since the faradic activity appears reliable even after five times, and the relative standard deviation (RSD) of the cathodic current peak of ampicillin nanoparticles in blood medium at GCE is $\pm 0.038 \%$ as shown in Table 1 . Table 2 shows the reliability of GCE for the anodic current peak of ampicillin nanoparticles in blood medium the RSD is \pm $0.52 \%$.

Fig. 7 shows the cyclic voltammogram of redox current peaks for ampicillin nanoparticles at five times which illustrated the good stability of the cyclic voltammogram of the GCE by overlapping the lines [20].

Table 1 Reliability of GCE as working electrode at SR is $100 \mathrm{mV} / \mathrm{s}$ for cathodic current peak of ampicillin trihydrate nanoparticles in blood medium

\begin{tabular}{cccc}
\hline Number & Ipc $(\mu \mathrm{A})$ & Mean & RSD $(\%)$ \\
\hline 1 & 38.1 & 38.5 & \\
2 & 38.5 & & \\
3 & 38.6 & & \\
4 & 38.7 & & \\
5 & 38.8 & & 0.038 \\
\hline
\end{tabular}


Table 2 Reliability of GCE as working electrode at SR is $100 \mathrm{mV} / \mathrm{s}$ for anodic current peak of ampicillin trihydrate nanoparticles in blood medium

\begin{tabular}{cccc}
\hline Number & Ipc $(\mu \mathrm{A})$ & Mean & RSD $(\%)$ \\
\hline 1 & 30.0 & 30.2 & \\
2 & 30.1 & & \\
3 & 30.2 & & \\
4 & 30.3 & & \\
5 & 30.4 & & 0.52 \\
\hline
\end{tabular}

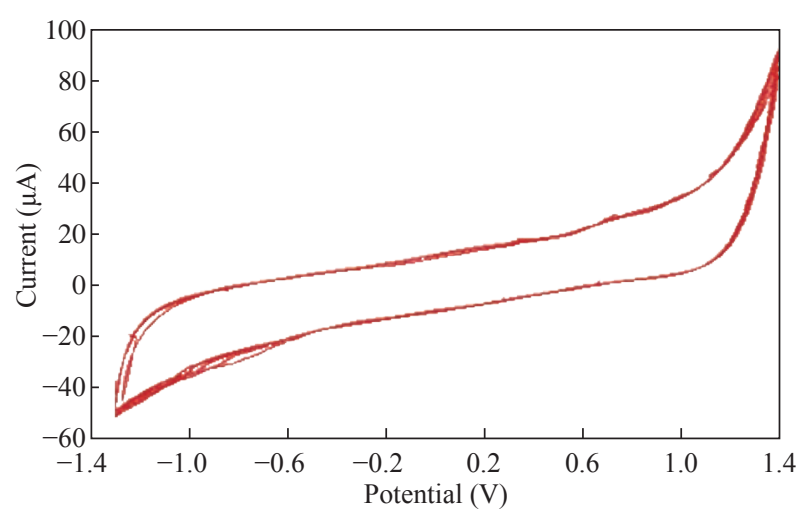

Fig. 6 Cyclic voltammogram of ampicillin nanoparticles in blood medium at five cycles on $\mathrm{GCE}$ versus $\mathrm{Ag} / \mathrm{AgCl}$.

\section{Effect of AA on ampicillin nanoparticles in blood medium}

Ampicillin trihydrate nanoparticles in blood medium with the presence of AA as an anti-oxidative reagent were studied at GCE which enhanced the oxidation current peak of the ampicillin nanoparticles and shifted to a higher potential. It was shown that increasing the concentration of AA enhanced the oxidation current peak of ampicillin nanoparticles (Fig. 7) and decreased the reduction current peak (Fig. 8). When plotting the oxidation-reduction current peaks of ampicillin nanoparticles against the concentration of AA, the linear relationship was $\mathrm{Y}=13.179 \mathrm{X}+35.086$, with $\mathrm{R}^{2}$

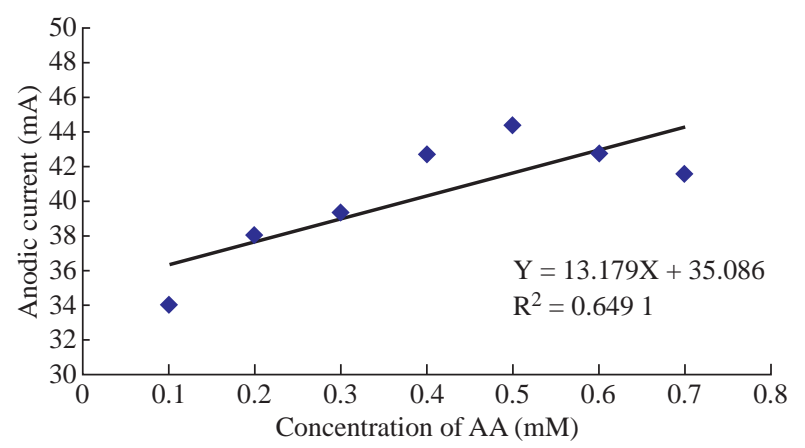

Fig. 7 Plot of oxidation current peak of ampicillin nanoparticles affected by different concentrations of AA in blood medium at $\mathrm{GCE}$ versus of $\mathrm{Ag} / \mathrm{AgCl}$.

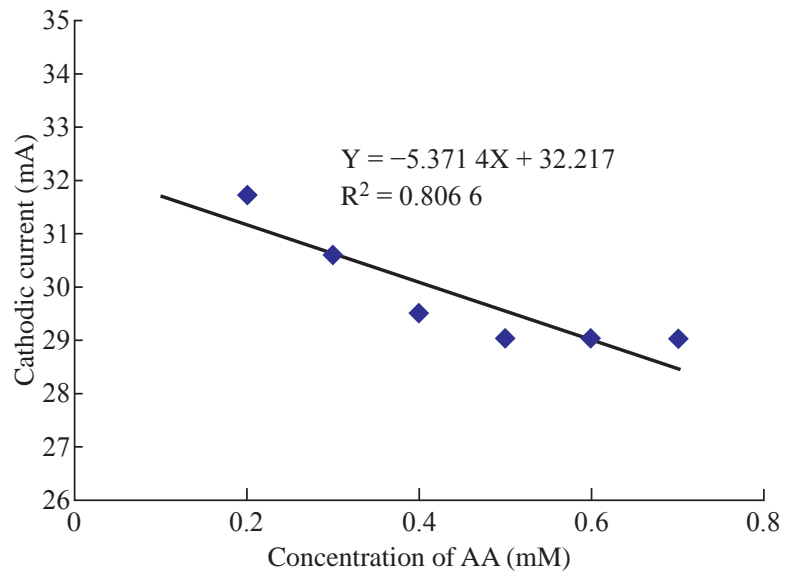

Fig. 8 Plot of reduction current peak of ampicillin nanoparticles affected by different concentrations of AA in blood medium at $\mathrm{GCE}$ versus of $\mathrm{Ag} / \mathrm{AgCl}$.

$=0.6491$ and $\mathrm{Y}=-5.314 \mathrm{X}+32.217$, with $\mathrm{R}^{2}=0.8066$ respectively. Hence, AA acted as electro-catalyst for the effect of ampicillin nanoparticles in blood medium by enhancing the biological activity against to the bacteria [21-24].

\section{Scanning electron microscopy}

Scanning electron microscopy (SEM) was used to examine the morphology of ampicillin trihydrate nanoparticles in blood medium as shown in Fig. 9. The nanoparticles of ampicillin illustrated in the SEM with below $100 \mathrm{~nm}$ dimension. Hence, the deep freezing method for convert micro- to nano dimension was successfully done.

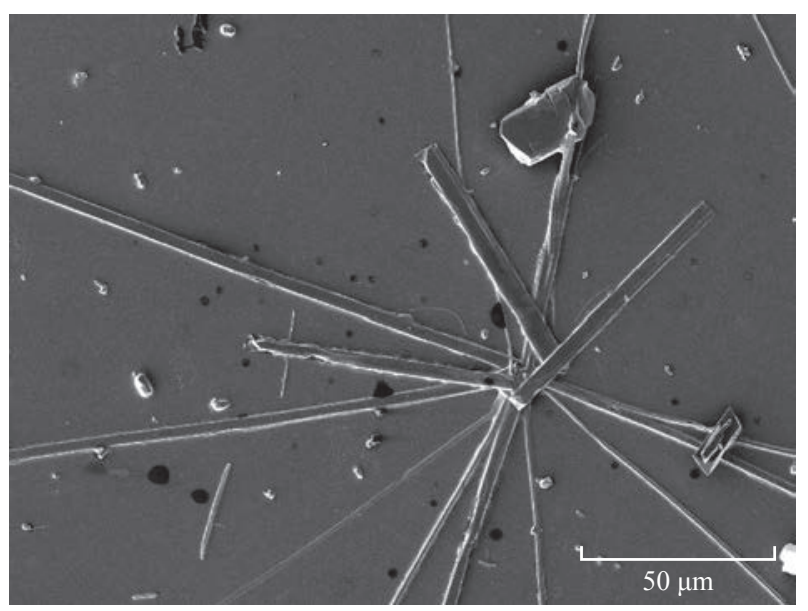

Fig. 9 SEM of ampicillin trihydrate nanoparticles.

\section{Conclusions}

Ampicillin was studied in the forms of microand nanoparticles in blood medium using cyclic voltammetric technique. The results showed that the ampicillin nanoparticles acted as a good anti-oxidative 
antibiotic in blood medium, while the ampicillin microparticles acted as an oxidative reagent with its oxidation current peak appearing at $1 \mathrm{~V}$ in blood medium. It was found that the effect of AA on the redox current peaks of ampicillin nanoparticles in blood medium as electro-catalyst could enhance the oxidation current peak and reduce the reduction current peak; hence, we considered ampicillin nanoparticles as a good and safe antibiotic in blood medium.

\section{Conflict of Interests}

The authors declare that no competing interest exists.

\section{References}

[1] M.M. Radhi, Y.K. Abdul-Amir, M.S. Khalaf, Electrochemical Effect of Acetylsalicylic Acid (Aspirin) in Present of Each Ascorbic Acid (AA) and Folic Acid (FA) in Normal Saline and Human Blood Samples, AASCIT Communi., 2016, 3(3): 152-159.

[2] M.M. Radhi, N.K. Al-Damlooji, B.K. Abed, Electrochemical Sensors for Detecting Mn (II) in Blood Medium. Sensors \& Transducers, 2013, 149: 89-93.

[3] S.M.M. Al-Mutoki, A.G. Wadday, and A.A. Abdullah, Effect of nano $\mathrm{TiO}_{2}$ dopant on electrical properties of SR8100/nano $\mathrm{TiO}_{2}$ PMNC. Results Phys., 2016, 6: 551553.

[4] M.M. Radhi, H.N. Abdullah1, M.S. Jabir et al., Electrochemical effect of ascorbic acid on redox current peaks of $\mathrm{CoCl}_{2}$ in blood medium. Nano Biomed. Eng., 2017, 9 (2): 103-106.

[5] M.M. Radhi, H.N. Abdullah, S.A. Al-Asadi, et al., Electrochemical oxidation effect of ascorbic acid on mercury ions in blood sample using cyclic voltammetry, Int J Ind Chem.,2016, 6 (4): 311-316.

[6] M.M. Radhi, H.N. Abdullah, N.M. Al-Shkir, Electrochemical study of the effect of ascorbic acid on redox current peaks of paracetamol in blood sample, Ir $J$ Biotech., 2015, 14: 63-70

[7] H. Meruvu, M. Vangalapati, S. Chaitanya, Synthesis and characterization of zinc oxide nanoparticles and its antimicrobial activity against bacillus subtilis and escherichia coli. Rasayan J Chem., 2011, 4: 217-222.

[8] A.N. Brown, K. Smith, T.A. Samuels, et al., Nanoparticles Functionalized with Ampicillin Destroy MultipleAntibiotic-Resistant Isolates of Pseudomonas aeruginosa and Enterobacter aerogenes and Methicillin-Resistant Staphylococcus aureus, Appl Environ Microbiol., 2012, 78(8): 2768-2774.

[9] E. Fattal, J. Rojas, L. Roblot-Treupel, Ampicillinloaded liposomes and nanoparticles: comparison of drug loading, drug release and in vitro antimicrobial activity. $J$ Microencapsulation, 1991, 8: 29-36.

[10] F. Forestier, P. Gerrier, C. Chaumanrd, et al., Effect of nanoparticle-bound ampicillin on the survival of Listeria monocytogenes in mouse peritoneal macrophages. $J$ Antimicrob Chemother (1992) 30 (2): 173-179.

[11] N. Esfandiari, S.M. Ghoreishi, Kinetics modeling of ampicillin nanoparticles synthesis via supercritical gas antisolvent process. J Supercritical Flu., 2013, 8: 119127.

[12] P. Saha, A.K. Goyal, G. Rath, Formulation and Evaluation of Chitosan-Based Ampicillin Trihydrate Nanoparticles. Tropical PharmaRes., 2010, 9 (5): 483-488.

[13] P. Lingwal, G.K. Bhatt, P. Kothiya, Formulation and evaluation of ampicillin loaded nanoparticles, IAJPS. 2015, 2 (9): 1289-1297.

[14] W. Abdelwahed, G. Degobert, S. Stainmesse and H. Fessi, Freeze-drying of nanoparticles: formulation, process and storage considerations. Adva drug deliv revi.,2006, 58(15):1688-1713.

[15] G.G. Jobitha, S.R. Kumar, G. Annadurai, Preparation and characterization of fruit mediated silver nanoparticles using pomegranate extract and assessment of its antimicrobial activities. J Environ Nanotechnol., 2013, 2 (1): 4-10.

[16] S.M.M. Al-Mutoki, B.A.K. Al-Ghzawi, A.A. Abdullah, et al., Synthesis and characterization of new epoxy/titanium dioxide nanocomposite. Nano Biomed. Eng., 2015, 7 (4): 135-138.

[17] H. Sardarabadi, M. Mashreghi, K. Jamialahmadi, T. Dianat, Resistance of nanobacteria isolated from urinary and kidney stones to broad-spectrum antibiotics. Iran $J$ Microbiol., 2014, 6 (4): 230-233.

[18] J. Sochor, J. Dobes, O. Krystofova, et al., Electrochemistry as a Tool for Studying Antioxidant Properties. Int. J. Electrochem. Sci., 2013. 8: 8464-8489.

[19] S. Sezgin, M. Ates, A. Elif, Scan Rate Effect of 1-(4-methoxyphenyl)-1H-Pyrrole Electro-coated on Carbon Fiber: Characterization via Cyclic Voltammetry, FTIR-ATR and Electrochemical Impedance Spectroscopy, Int. J. Electrochem. Sci., 2012, 7: 1093-1106.

[20] M.M. Rahman, A.J. Ahammad, j. Joon-Hyung, Comprehensive Review of Glucose Biosensors Based on Nanostructured Metal-Oxides, Sensors 2010, 10: 48554886.

[21] A.A. Albakry, A.M. Jassim, S.A. Alassady,. Electrochemical study of $\mathrm{Pb}(\mathrm{II})$ in present of each ascorbic acid, glucose, urea and uric acid using blood medium as an electroly. Nano Biomed. Eng., 2016, 8: 9-15.

[22] S.M.M. Al-Mutoki,m, B.A. Al-Ghzawi, S. AbdulAmohsin, Enhancement of mechanical properties of polyamide hexaglycol by dispersion of $\mathrm{TiO}_{2}$ nanofiller. Nano Biomed. Eng., 2016, 8(2): 55-59.

[23] K.W. Al-Janabi, Extraction of cobalt(II) from aqueous solution by $N, N^{\prime}$-carbonyl difatty amides. Chin Chem Let., 2011, 22(4): 469-472.

[24] M.M. Radhi, H.A.T. Al-Shimmari, E.A.J. Al-Mulla, et al., New Voltammetric Study of $\mathrm{MgCl}_{2}$ as Alternative Contrast Media in MRI Molecular Imaging. Nano Biomed. Eng., 2017, 9(2): 152-161.

Copyright@ 2017 Muhammed Mizher Radhi, Zuhair Numan Hamed, Selda Sabah Ezzaldeen, and Emad Abbas Jaffar AlMulla. This is an open-access article distributed under the terms of the Creative Commons Attribution License, which permits unrestricted use, distribution, and reproduction in any medium, provided the original author and source are credited. 